\title{
Dimensional crossover of the dephasing time in disordered mesoscopic rings
}

\author{
M. Treiber, ${ }^{1}$ O. M. Yevtushenko, ${ }^{1}$ F. Marquardt, ${ }^{1}$ J. von Delft, ${ }^{1}$ and I. V. Lerner ${ }^{2}$ \\ ${ }^{1}$ Arnold Sommerfeld Center and Center for Nano-Science, Ludwig-Maximilians-University, Munich D-80333, Germany \\ ${ }^{2}$ School of Physics and Astronomy, University of Birmingham, Birmingham B15 2TT, United Kingdom
}

(Received 13 October 2009; published 10 November 2009)

\begin{abstract}
We study dephasing by electron interactions in a small disordered quasi-one-dimensional (1D) ring weakly coupled to leads. We use an influence functional for quantum Nyquist noise to describe the crossover for the dephasing time $\tau_{\varphi}(T)$ from diffusive or ergodic $1 \mathrm{D}\left(\tau_{\varphi}^{-1} \propto T^{2 / 3}, T^{1}\right)$ to zero-dimensional (0D) behavior $\left(\tau_{\varphi}^{-1} \propto T^{2}\right)$ as $T$ drops below the Thouless energy. The crossover to $0 \mathrm{D}$, predicted earlier for two-dimensional and three-dimensional systems, has so far eluded experimental observation. The ring geometry holds promise of meeting this long-standing challenge, since the crossover manifests itself not only in the smooth part of the magnetoconductivity but also in the amplitude of Altshuler-Aronov-Spivak oscillations. This allows signatures of dephasing in the ring to be cleanly extracted by filtering out those of the leads.
\end{abstract}

DOI: $10.1103 /$ PhysRevB.80.201305

\section{INTRODUCTION}

Over the last 20 years numerous theoretical ${ }^{1-8}$ and experimenta ${ }^{10-15}$ works have studied the mechanism of dephasing in electronic transport and its dependence on temperature $T$ and dimensionality in disordered condensedmatter systems. At low temperatures dephasing is mainly due to electron interactions, with the dephasing time $\tau_{\varphi}(T)$ increasing as $T^{-a}$ when $T \rightarrow 0$.

The dephasing time controls the scale of a negative weak localization (WL) correction to the magnetoconductivity and (under certain conditions) the magnitude of universal conductance fluctuations (UCFs). If $T$ is so low that $\tau_{\varphi}$ exceeds $\tau_{\mathrm{Th}}=\hbar / E_{\mathrm{Th}}$, the time required for an electron to cross (diffusively or ballistically) a mesoscopic sample ( $E_{\mathrm{Th}}$ is the Thouless energy), UCFs become $T$ independent. This leaves WL as the only tool to measure the $T$ dependence of dephasing in mesoscopic wires or quantum dots at very low $T$. For quantum dots, a dimensional crossover was predicted ${ }^{4}$ from $\tau_{\varphi} \propto T^{-1}$, typical for a two-dimensional (2D) electron gas, ${ }^{1}$ to $\tau_{\varphi} \propto T^{-2}$ when the temperature is lowered into the zerodimensional (0D) regime,

$$
\hbar / \tau_{\varphi} \ll T \ll E_{\mathrm{Th}},
$$

where the coherence length and the thermal length are both larger than the system size independent of geometry and real dimensionality of the sample. Although the $\tau_{\varphi} \propto T^{-2}$ behavior is quite generic, arising from the fermionic statistics of conduction electrons, experimental efforts ${ }^{13}$ to observe it have so far been unsuccessful. The reasons for this are unclear. Conceivably dephasing mechanisms other than electron interactions were dominant, or the regime of validity of the $0 \mathrm{D}$ description had not been reached. In any case, other ways of testing the dimensional crossover for $\tau_{\varphi}$ are desirable.

Here we study dephasing in a quasi-1D mesoscopic ring weakly coupled to two well-conducting leads through narrow point contacts. We find a dimensional crossover for $\tau_{\varphi}(T)$ from diffusive or ergodic 1D $\left(\propto T^{-2 / 3}, T^{-1}\right)$ to $0 \mathrm{D}\left(\propto T^{-2}\right)$ behavior as $T$ is decreased below $E_{\mathrm{Th}}$ and propose a detailed experimental scenario for observing this behavior. It reveals itself not only via the WL corrections to the smooth part of magnetoconductivity but also via the amplitude of the
PACS number(s): 73.63.-b, 72.10.-d, 72.15.Rn

Altshuler-Aronov-Spivak (AAS) oscillations ${ }^{16}$ that result from closed trajectories with a nonzero winding number acquiring the Aharonov-Bohm phase. For sufficiently weak lead-ring coupling (specified below), the magnitude of AAS oscillations will be independent of dephasing in the leads. Thus, the ring geometry provides a more promising setup for the observation of the dimensional crossover than $2 \mathrm{D}$ or $3 \mathrm{D}$ settings.

\section{DEPHASING IN WEAK LOCALIZATION}

The WL correction to the conductivity is governed by coherent backscattering of the electrons from static disorder and, to the lowest order, is due to the enhancement of the return probability caused by constructive interference of two time reversed trajectories described by the so-called Cooperon $\mathcal{C} .{ }^{17,18}$ In this order, the WL correction to the conductivity, in units of the Drude conductivity $\sigma_{0}$, is given by ${ }^{19}$

$$
\Delta g=\frac{\Delta \sigma}{\sigma_{0}}=-\frac{1}{\pi \nu} \int_{0}^{\infty} d t^{\prime} \mathcal{C}\left(t^{\prime}\right) .
$$

Here $\nu$ is the electron density of states per spin at the Fermi surface and $\hbar=1$ henceforward. Dephasing limits the scale of this contribution and effectively results in the suppression of the Cooperon at long times:

$$
\mathcal{C}(t) \equiv \mathcal{C}_{0}(t) \exp \left[-t / \tau_{H}-t / \tau_{\mathrm{dw}}-\mathcal{F}(t)\right] .
$$

We consider here low temperatures where the phonon contribution to dephasing is negligible and three main sources contribute to the Cooperon decay with time: an applied magnetic field $H$ characterized by the time scale $\tau_{H}$ (Ref. 20); the leakage of particles from the ring characterized by the dwell time $\tau_{\mathrm{dw}}$ (Ref. 21); and electron interactions, whose effects can be described in terms of the decay function $\mathcal{F}(t),{ }^{1,3}$ which grows with time and may be used to define a dephasing time via $\mathcal{F}\left(\tau_{\varphi}\right)=1$.

$\mathcal{F}(t)$ can be obtained using the influence functional approach, ${ }^{6,7}$ which gives results for the magnetoconductivity that are practically equivalent to those originally obtained in Ref. 1. Roughly speaking, an electron traversing a random walk trajectory $x\left(t_{1}\right)$ of duration $t$ acquires a random phase 
$\varphi_{t}=\int_{0}^{t} d t_{1} V\left(x\left(t_{1}\right), t_{1}\right)$ due to the random potential $V$ describing the Nyquist noise originating from electron interactions; the variance of this phase, averaged over all closed random walks (crw), gives the decay function, $\mathcal{F}(t)=\frac{1}{2}\left\langle\delta \varphi_{t}^{2}\right\rangle_{\text {crw }}$. A careful treatment ${ }^{6,7}$ gives

$$
\mathcal{F}(t)=\int_{0}^{t} d^{2} t_{1,2}\left\langle\overline{V V}\left(x_{12}, t_{12}\right)-\overline{V V}\left(x_{12}, \bar{t}_{12}\right)\right\rangle_{\text {crw }},
$$

where $x_{12} \equiv x\left(t_{1}\right)-x\left(t_{2}\right) ; t_{12} \equiv t_{1}-t_{2} ; \bar{t}_{12} \equiv t_{1}+t_{2}-t$. The noise is assumed Gaussian, with correlation function

$$
\overline{V V}(x, t)=\left(2 e^{2} T / \sigma_{0} A\right) Q(x) \delta_{T}(t) .
$$

Here $A$ is the wire's cross-sectional area, the diffuson $Q(x)$ is the time-averaged solution of the diffusion equation, and $\delta_{T}(t)$ is a broadened $\delta$ function of width $T \simeq 1 / T$ and height $\simeq T$ given by $^{7,8}$

$$
\delta_{T}(t)=\pi T w(\pi T t), \quad w(y)=\frac{y \operatorname{coth}(y)-1}{\sinh ^{2}(y)} .
$$

This form takes into account the Pauli principle in a quantum description of Nyquist noise and reproduces the results ${ }^{5}$ of leading order perturbation theory in the interaction for $\Delta g$. The broadening of $\delta_{T}(t)$ is the central difference between quantum noise and the classical noise considered in previous treatments, ${ }^{1,6}$ which used a sharp $\delta(t)$ function instead. Note that Eq. (4) is free from IR singularities, because the $x$-independent part of $\overline{V V}$ (the diffuson "zero mode") does not contribute to $\mathcal{F}$.

\section{QUALITATIVE PICTURE}

We begin with a qualitative discussion of dephasing in an isolated quasi-1D system of size $L$. Since Nyquist electric field fluctuations are white noise in space, the $x$ dependence of $V$ behaves like a random walk in space $(\sim \sqrt{|x|})$, so that $Q(x) \sim|x|$. For $\tau_{T} \ll \tau_{\mathrm{Th}}$, the potential seen during one traversal of the system is also white noise in time, i.e., $\delta_{T}(t) \rightarrow \delta(t)$.

In the diffusive regime $\left(\tau_{T} \ll t \ll \tau_{\mathrm{Th}}\right)$, a random walk $x\left(t_{1}\right)$ of duration $t$ does not feel the boundaries, hence, $\left|x\left(t_{1}\right)\right| \sim \sqrt{t_{1}}$. Thus, $\mathcal{F}(t) \propto T \int_{0}^{t} \sqrt{t_{1}} d t_{1} \propto T t^{3 / 2}$, reproducing the well-known result $\tau_{\varphi} \propto T^{-2 / 3}$. ${ }^{2}$

In the ergodic regime $\left(\tau_{T} \ll \tau_{\mathrm{Th}} \ll t\right)$, the trajectory fully explores the whole system, thus, $\left|x\left(t_{1}\right)\right| \sim L$ instead, which reproduces $\mathcal{F}(t) \propto T L t$ and $\tau_{\varphi} \propto T^{-1}$.

We are primarily interested in the $0 \mathrm{D}$ regime reached at $T \ll E_{\mathrm{Th}}\left(\tau_{\mathrm{Th}} \ll \tau_{T} \ll t\right)$. In contrast to the previous two regimes, a typical trajectory visits the vicinity of any point $x$ in the interval $[0, L]$ several times during the time $\tau_{T}$ (see Fig. 1). On time scales shorter than this time $\tau_{T}$ the potential is effectively frozen, so that the broadened $\delta$ function in Eq. (5) saturates at its maximum, $\delta_{T}(t) \rightarrow T$, and the variance of $V$ is of order $T^{2}|x|$. The phase picked up during $\tau_{T}$ becomes $\varphi_{\tau_{T}}=\tau_{T} \int_{0}^{L} d x p(x) V\left(x, t_{1}\right)$, where $p(x) d x$ is the fraction of time the trajectory spends near $x$. Then only small statistical deviations from the completely homogeneous limit, $p(x)=1 / L$ (reached for $\tau_{T} \rightarrow \infty$ ), yield a phase difference between the two time-reversed trajectories. These deviations scale like

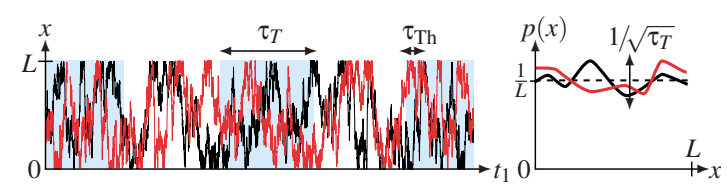

FIG. 1. (Color online) Left: a pair of time-reversed diffusive trajectories exploring ergodically a region of size $L$. The fluctuating noise potential is frozen during time intervals (indicated by shading) of duration $\tau_{T}=1 / T$ sketched here to be $\gg \tau_{\mathrm{Th}}$. Right: the density $p(x)$ of points $x$ visited by a particular trajectory during the time interval $\tau_{T}$ fluctuates around $1 / L$ with fluctuations $\delta p \sim 1 / \sqrt{\tau_{T}}$.

$\delta p \sim 1 / \sqrt{\tau_{T}}$, since the number of "samples" (i.e., of traversals of the system during time $\tau_{T}$ ) effectively grows with $\tau_{T}$. Thus, setting $V \sim T \sqrt{|x|}$, we estimate $\varphi_{\tau_{T}} \sim \tau_{T} L^{3 / 2} T / \sqrt{\tau_{T}}$, so that $\left\langle\delta \varphi_{\tau_{T}}^{2}\right\rangle \sim L^{3} T^{2} \tau_{T}$. Adding up the contributions from $t / \tau_{T}$ independent time intervals $\left(t \gg \tau_{T}\right)$, we find $\mathcal{F}(t) \propto L^{3} T^{2} t$, implying $\tau_{\varphi} \sim T^{-2}$, characteristic of $0 \mathrm{D}$ systems. ${ }^{4}$ Thus, when $\tau_{\mathrm{Th}}$ becomes the smallest time scale, a dimensional crossover occurs and the system becomes effectively $0 \mathrm{D}$.

The qualitative behavior of $\tau_{\varphi}$ in all three regimes also follows upon extracting $\tau_{\varphi}$ self-consistently from the standard perturbative expression for the Cooperon self-energy. ${ }^{2,7,8}$ Inserting the usual cutoffs $T$ and $1 / \tau_{\varphi}$ for the frequency transferred between the diffusive electrons and their Nyquist noise environment and excluding the diffuson zeroth mode via a cutoff at $1 / L$ of the transferred momentum, we have (omitting numerical prefactors)

$$
\frac{1}{\tau_{\varphi}} \propto \frac{T}{g_{1} L} \int_{1 / \tau_{\varphi}}^{T} d \omega \int_{1 / L}^{\infty} \frac{D d q}{\left(D q^{2}\right)^{2}+\omega^{2}}, \quad g_{1}=\frac{h \sigma_{0}}{e^{2}} \frac{A}{L},
$$

where $g_{1}$ is the $1 \mathrm{D}$ dimensionless conductance, $D=v_{F} l$ the $1 \mathrm{D}$ diffusion constant, $v_{F}$ the Fermi velocity, and $\ell$ the mean free path. Writing $E_{\mathrm{Th}}=D / L^{2}$ this yields $\tau_{\varphi} \propto\left(g_{1} / \sqrt{E_{\mathrm{Th}}} T\right)^{2 / 3}$, $g_{1} / T$, or $E_{\mathrm{Th}} g_{1} / T^{2}$ for the diffusive $\left(\tau_{T} \ll \tau_{\varphi} \ll \tau_{\mathrm{Th}}\right)$, ergodic $\left(\tau_{T} \ll \tau_{\mathrm{Th}} \ll \tau_{\varphi}\right)$, or 0D $\left(\tau_{\mathrm{Th}} \ll \tau_{T} \ll \tau_{\varphi}\right)$ regimes, respectively, as above (with dimensionful parameters reinstated). Equation (7) illustrates succinctly that the modes dominating dephasing lie near the infrared cutoff $\left(\omega \simeq \tau_{\varphi}^{-1}\right.$ or $\left.E_{\mathrm{Th}}\right)$ for the diffusive or ergodic regimes but near the ultraviolet cutoff $\omega \simeq T$ for the OD regime [which is why, in the latter, the broadening of $\delta_{T}(t)$ becomes important].

\section{ANALYTICAL RESULTS}

We now turn to a quantitative analysis. ${ }^{22}$ The diffuson in the ring geometry is ${ }^{18}$

$$
Q(x)=\frac{L_{\mathrm{dw}}}{2} \frac{\cosh \left([L-2|x|] / 2 L_{\mathrm{dw}}\right)}{\sinh \left(L / 2 L_{\mathrm{dw}}\right)},
$$

where $L_{\mathrm{dw}}=\sqrt{D \tau_{\mathrm{dw}}}$ and $x$ is the cyclic coordinate along the ring. Terms of order $\tau_{\mathrm{Th}} / \tau_{\mathrm{dw}}$ (small for an almost isolated ring) do not change the parametric dependence of $\mathcal{F}$ on $T, t$, and $L$ so that we neglect them below, setting $\tau_{\mathrm{dw}}=\infty$ in Eq. (4). Inserting Eqs. (5), (6), and (8) into Eq. (4), the decay function $\mathcal{F}_{n}(t)$ for a given winding number $n$ can be calculated as in Ref. 6 but replacing $\delta(t)$ by $\delta_{T}(t)$ : 


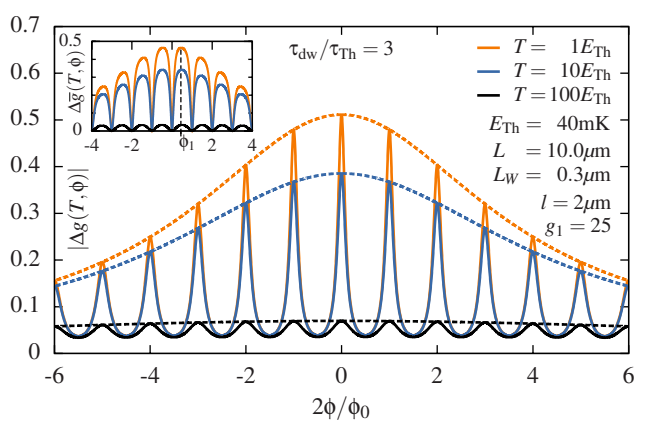

FIG. 2. (Color online) The WL correction $|\Delta g(T, \phi)|$ (solid lines), its envelope $\left|\Delta g_{\text {en }}(T, \phi)\right|$ (dashed lines), and their difference $\Delta \bar{g}=\left|\Delta g_{\text {en }}\right|-|\Delta g|$ (inset), plotted as function of magnetic flux $2 \phi / \phi_{0}$, for three different temperatures.

$$
\begin{gathered}
\mathcal{F}_{n}(t)=-\frac{4 \pi T t}{g_{1} L} \int_{0}^{1} d u z(u)\langle Q\rangle_{\mathrm{crw}}(u), \\
\langle Q\rangle_{\mathrm{crw}}(u)=\frac{L}{2} \sum_{k=1}^{\infty} \frac{\cos (2 \pi k n u)}{(\pi k)^{2}} e^{-(2 \pi k)^{2} E_{\mathrm{Th}} t u(1-u)}, \\
z(u)=-2 \pi T t(1-u) w(\pi T t u)+\int_{-\pi T t u}^{\pi T t u} d v w(v) .
\end{gathered}
$$

Equation (9) yields the following results for the diffusive, ${ }^{1,23}$ ergodic, ${ }^{6}$ and $0 \mathrm{D}$ regimes at $|n| \ll E_{\mathrm{Th}} t$ :

$$
\mathcal{F}_{n}(t) \simeq\left\{\begin{array}{cc}
\frac{\delta_{n, 0} \pi^{3 / 2}}{2 g_{1}} \sqrt{E_{\mathrm{Th}}} T t^{3 / 2}, & \tau_{T} \ll t \ll \tau_{\mathrm{Th}} \\
\frac{\pi T t}{3 g_{1}}, & \tau_{T} \ll \tau_{\mathrm{Th}} \ll t \\
\frac{\pi^{2}}{270 g_{1}} \frac{T^{2} t}{E_{\mathrm{Th}}}, & \tau_{\mathrm{Th}} \ll \tau_{T} \ll t .
\end{array}\right.
$$

Subleading terms in the three limiting cases (10a)-(10c) are of order $\mathcal{O}\left[\left(\tau_{T} / t\right)^{1 / 2},\left(t / \tau_{\mathrm{Th}}\right)^{1 / 2}\right], \mathcal{O}\left[\left(\tau_{T} / \tau_{\mathrm{Th}}\right)^{1 / 2}, \tau_{\mathrm{Th}} / t\right]$, and $\mathcal{O}\left[\left(\tau_{\mathrm{Th}} / \tau_{T}\right)^{2},\left(\tau_{T} / t\right)\right]$, respectively. Note that the crossover temperatures where $\tau_{\varphi}^{\text {diff }} \simeq \tau_{\varphi}^{\text {erg }}$ or $\tau_{\varphi}^{\text {erg }} \simeq \tau_{\varphi}^{0 \mathrm{D}}$, namely, $c_{1} g_{1} E_{\mathrm{Th}}$ or $c_{2} E_{\mathrm{Th}}$, respectively, involve large prefactors, $c_{1}=27 / 4 \simeq 7$ and $c_{2}=90 / \pi \simeq 30$, which should aid experimental efforts to reach the $0 \mathrm{D}$ regime.

For a ring of rectangular cross section $A=L_{W} L_{H}$ and circumference $L$, the Cooperon can then be written as

$$
\mathcal{C}(t) \simeq \sum_{n=-\infty}^{+\infty} \frac{e^{-(n L)^{2} / 4 D t}}{\sqrt{4 \pi D t}} e^{-t / \tau_{H^{-}} \mathcal{F}_{n}(t)-t / \tau_{\mathrm{dw}}} e^{i n \theta},
$$

with (restoring $\hbar) \tau_{H}=9.5(\hbar c / e H)^{2} \times\left(l / D L_{W}^{3}\right)$ and $\theta$ $=4 \pi \phi / \phi_{0}$, where $\phi=\pi(L / 2 \pi)^{2} H$ is the flux through the ring and $\phi_{0}=h c / e .^{15,16,24}$ Inserting Eqs. (9) and (11) into Eq. (2) gives the desired WL correction for the ring weakly coupled to leads. The resulting value of $|\Delta g(T, \phi)|$ increases with decreasing $T$, Fig. 2, in a manner governed by $\tau_{\varphi}$ : since only trajectories with $|n| \lesssim 2 \sqrt{t / \tau_{\mathrm{Th}}}$ contribute, the diffusive regime ( $n$ restricted to 0 ) gives $|\Delta g| \propto \sqrt{\tau_{\varphi} / \tau_{\mathrm{Th}}} / g_{1} \propto\left(E_{\mathrm{Th}} / g_{1}^{2} T\right)^{1 / 3}$, whereas the ergodic regime (sum on $n$ is $\propto t^{1 / 2}$ ) gives $|\Delta g| \propto\left(\tau_{\varphi} / \tau_{\mathrm{Th}}\right) / g_{1} \propto E_{\mathrm{Th}} / T$, as long as $\tau_{\varphi}<\tau_{H}, \tau_{\mathrm{dw}}$. With decreasing $T$, the growth of $|\Delta g(T, \phi)|$

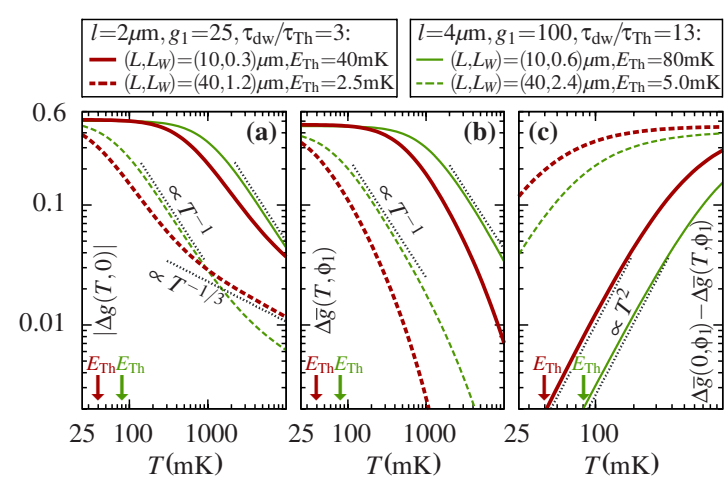

FIG. 3. (Color online) $T$ dependence of (a) the WL correction at zero field, $|\Delta g(T, 0)|$ and (b) at finite field with envelope subtracted, $\Delta \bar{g}\left(T, \phi_{1}\right)$; (c) the difference $\Delta \bar{g}\left(0, \phi_{1}\right)-\Delta \bar{g}\left(T, \phi_{1}\right)$, which reveals a crossover to $T^{2}$ behavior for $T \ll 30 E_{\mathrm{Th}}$. The flux $\phi_{1}$, which weakly depends on $T$, marks the first maximum of $\Delta \bar{g}(T, \phi)$; see inset of Fig. 2.

saturates toward $|\Delta g(0, \phi)|$ once $\tau_{\varphi}$ increases past $\min \left(\tau_{H}, \tau_{\mathrm{dw}}\right)$, with $|\Delta g(0, \phi)|-|\Delta g(T, \phi)| \propto \tau_{\varphi}^{-1}$ vanishing as $T$ or $T^{2}$ in the ergodic or $0 \mathrm{D}$ regimes, respectively.

\section{FILTERING OUT LEADS}

For simplicity, above we did not model the leads explicitly. In real experiments, however, $\Delta g$ is affected by dephasing in the leads, which might mask the signatures of dephasing in the ring. Similar concerns apply to quantum dots connected to leads (cf. the $\tau_{\varphi} \propto T^{-1}$ behavior observed in Ref. 13), or finite-size effects in a network of disordered wires, ${ }^{15}$ where paths encircling a given unit cell might spend significant time in neighboring unit cells as well (cf. $T^{-1 / 3}$ behavior observed in Refs. 15 and 25 at $\tau_{\varphi} / \tau_{\mathrm{Th}} \geq 1$ ). To filter out the effect of leads, we construct ${ }^{15}$ from $|\Delta g(T, \phi)|$ its nonoscillatory envelope $\left|\Delta g_{\text {en }}(T, \phi)\right|$, obtained by setting $\theta=0$ in Eq. (11) while retaining $\tau_{H} \neq 0$, and study the difference

$$
\Delta \bar{g}(T, \phi)=\left|\Delta g_{\text {en }}(T, \phi)\right|-|\Delta g(T, \phi)| .
$$

This procedure is illustrated in Fig. 2. $\Delta \bar{g}$ is dominated by paths with winding numbers $n \geq 1$ which belong to the ring. Contributions to $\Delta \bar{g}$ from Cooperons extending over both the ring and a lead will be subleading for well-conducting leads with a small contact-lead-contact return probability. Concretely, for $N$-channel point contacts with conductance $g_{\text {cont }}=N T_{\text {cont }}$, this requires leads with dimensionless conductance $g_{\text {lead }} \gg N .^{26}$

\section{SUGGESTED EXPERIMENTS}

To observe the predicted 1D-to-0D crossover experimentally, several conditions need to be satisfied. Our theory assumes (i) $L \gg \ell \gg L_{W} \gg \lambda_{F}\left(\lambda_{F}\right.$ is the Fermi wavelength). Ensuring that we stay in the WL regime requires (ii) a large dimensionless conductance, $g_{1} \propto(\ell / L)\left(L_{W} L_{H} / \lambda_{F}^{2}\right) \gg 1$, and (iii) a finite $\tau_{\mathrm{dw}}$ to limit the growth of $\Delta g$ with decreasing $T$; choosing the limit, somewhat arbitrarily, as $\Delta g \lesssim \frac{1}{2}$ at $T, H$ $=0$ implies $\tau_{\mathrm{dw}} / \tau_{\mathrm{Th}} \lesssim g_{1} / 8$. Estimating $\tau_{\mathrm{dw}} / \tau_{\mathrm{Th}} \simeq g_{1} / g_{\text {cont }}$, this implies $8 \lesssim g_{\text {cont }}$ and thus the absence of Coulomb block- 
ade. (iv) We also need $\tau_{\mathrm{Th}} \ll \tau_{\mathrm{dw}}$, or $g_{\text {cont }} \ll g_{1}$, to ensure that trajectories with $|n| \geq 1$, responsible for AAS oscillations, remain relevant. (v) To maximize the WL signal, the transmission per channel should be maximal, thus, we suggest $T_{\text {cont }} \simeq 1$ and $N \simeq 10$. (vi) The relevant temperature range, $\left[T_{\mathrm{dil}}, T_{\mathrm{ph}}\right]$, is limited from below by dilution refrigeration $\left(T_{\mathrm{dil}} \simeq 10 \mathrm{mK}\right)$ and from above by our neglect of phonons $\left(T_{\mathrm{ph}} \simeq 5 \mathrm{~K}\right)$. (vii) The ring should be small enough that $c_{2} E_{\mathrm{Th}} \gtrsim T_{\mathrm{dil}}$. (viii) The interaction-induced dephasing rate $\tau_{\varphi}^{-1}$, though decreasing with decreasing $T$, should for $T \simeq T_{\text {dil }}$ not yet be negligible compared to the $T$-independent rates $\tau_{H}^{-1}$ and $\tau_{\mathrm{dw}}^{-1}$. These constraints can be met, e.g., with rings prepared from a $2 \mathrm{D} \mathrm{GaAs} / \mathrm{AlGaAs}$ heterostructure with $\lambda_{F} \approx 30 \mathrm{~nm}, v_{F} \approx 2.5 \times 10^{5} \mathrm{~m} / \mathrm{s}$, and $g_{1}=4 \pi L_{W} l / \lambda_{F} L$, by adjusting $g_{1}$ and $E_{\mathrm{Th}}$ by suitably choosing $L$ and $L_{W}$.

To illustrate this, numerical results for $|\Delta g|$ and $\Delta \bar{g}$, obtained from Eq. (2) using experimentally realizable parameters, ${ }^{9,10,15,27}$ are shown in Figs. 2 and 3 for several combinations of $\ell, L$, and $L_{W}$. The regime where $\Delta g$ exhibits diffusive $T^{-1 / 3}$ behavior $\left(7 g_{1} E_{\mathrm{Th}} \ll T \ll T_{\mathrm{ph}}\right)$ is visible only for our smallest choices of both $g_{1}$ and $E_{\mathrm{Th}}$ [Fig. 3(a); heavy dashed line]. AAS oscillations in $|\Delta g|$ and $\Delta \bar{g}$ (Fig. 2), which require $\tau_{\mathrm{Th}} \ll \tau_{\varphi}$, first emerge at the crossover from the diffusive to the ergodic regime. They increase in magnitude with decreasing $T$, showing ergodic $T^{-1}$ behavior for $30 E_{\mathrm{Th}} \ll T \ll 7 g_{1} E_{\mathrm{Th}}$ [Figs. 3(a) and 3(b)], and eventually saturate toward their $T=0$ values, with $\Delta \bar{g}(0, \phi)-\Delta \bar{g}(T, \phi)$ showing the predicted OD behavior, $\propto T^{2}$, for $T \leqq 5 E_{\mathrm{Th}}$, see Fig. 3(c) (there $\tau_{\varphi} \gg \tau_{\mathrm{dw}}$, i.e., dephasing is weak).

\section{CONCLUSIONS}

The AAS oscillations of a quasi-1D ring weakly coupled to leads can be exploited to filter out the effects of dephasing in the leads, thus, offering a way to finally observe, for $T \lesssim 5 E_{\mathrm{Th}}$, the elusive but fundamental 0D behavior $\tau_{\varphi} \sim T^{-2}$. This would allow quantitative experimental tests of the role of temperature as ultraviolet frequency cutoff in the theory of dephasing.

\section{ACKNOWLEDGMENTS}

We acknowledge helpful discussions with B. Altshuler, C. Bäuerle, N. Birge, Y. Blanter, P. Brouwer, L. Glazman, Y. Imry, V. Kravtsov, J. Kupferschmidt, A. Mirlin, Y. Nazarov, A. Rosch, D. Weiss, and V. Yudson and support from the DFG through Grant No. SFB TR-12, the Emmy-Noether program, and the Nanosystems Initiative Munich Cluster of Excellence. We also acknowledge support from the NSF under Grant No. PHY05-51164 and from the EPSRC-GB under Grant No. T23725/01.
${ }^{1}$ B. L. Altshuler, A. G. Aronov, and D. E. Khmelnitsky, J. Phys. C 15, 7367 (1982).

${ }^{2}$ H. Fukuyama and E. Abrahams, Phys. Rev. B 27, 5976 (1983).

${ }^{3}$ B. L. Altshuler and A. G. Aronov, in Electron-Electron Interactions in Disordered Systems, edited by A. L. Efros and M. Pollak (North-Holland, Amsterdam, 1985), Vol. 1.

${ }^{4}$ U. Sivan, Y. Imry, and A. G. Aronov, Europhys. Lett. 28, 115 (1994).

${ }^{5}$ I. L. Aleiner, B. L. Altshuler, and M. E. Gershenson, Waves Random Media 9, 201 (1999); I. L. Aleiner, B. L. Altshuler, and Y. M. Galperin, Phys. Rev. B 63, 201401(R) (2001); I. L. Aleiner and Y. M. Blanter, ibid. 65, 115317 (2002).

${ }^{6}$ T. Ludwig and A. D. Mirlin, Phys. Rev. B 69, 193306 (2004); C. Texier and G. Montambaux, ibid. 72, 115327 (2005).

${ }^{7}$ F. Marquardt, J. von Delft, R. A. Smith, and V. Ambegaokar, Phys. Rev. B 76, 195331 (2007).

${ }^{8}$ J. von Delft, F. Marquardt, R. A. Smith, and V. Ambegaokar, Phys. Rev. B 76, 195332 (2007).

${ }^{9}$ Y. Niimi, Y. Baines, T. Capron, D. Mailly, F. Y. Lo, A. D. Wieck, T. Meunier, L. Saminadayar, and C. Bäuerle, Phys. Rev. Lett. 102, 226801 (2009).

${ }^{10}$ C. M. Marcus, R. M. Westervelt, P. F. Hopkins, and A. C. Gossard, Phys. Rev. B 48, 2460 (1993).

${ }^{11}$ A. Yacoby, M. Heiblum, H. Shtrikman, V. Umansky, and D. Mahalu, Semicond. Sci. Technol. 9, 907 (1994).

${ }^{12}$ B. Reulet, H. Bouchiat, and D. Mailly, Europhys. Lett. 31, 305 (1995).

${ }^{13}$ A. G. Huibers, M. Switkes, C. M. Marcus, K. Campman, and A. C. Gossard, Phys. Rev. Lett. 81, 200 (1998); A. G. Huibers, S. R. Patel, C. M. Marcus, P. W. Brouwer, C. I. Duruoz, and J. S. Harris, ibid. 81, 1917 (1998); A. G. Huibers, J. A. Folk, S. R. Patel, C. M. Marcus, C. I. Duruoz, and J. S. Harris, ibid. 83,
5090 (1999).

${ }^{14}$ A. B. Gougam, F. Pierre, H. Pothier, D. Esteve, and N. O. Birge, J. Low Temp. Phys. 118, 447 (2000).

${ }^{15}$ M. Ferrier, A. C. H. Rowe, S. Gueron, H. Bouchiat, C. Texier, and G. Montambaux, Phys. Rev. Lett. 100, 146802 (2008).

${ }^{16}$ B. L. Altshuler, A. G. Aronov, and B. Z. Spivak, JETP Lett. 33, 94 (1981).

${ }^{17}$ L. P. Gor'kov, A. I. Larkin, and D. E. Khmel'nitskii, JETP Lett. 30, 228 (1979).

${ }^{18}$ E. Akkermans and G. Montambaux, Mesoscopic Physics of Electrons and Photons (Cambridge University Press, Cambridge, 2007).

${ }^{19}$ D. E. Khmel'nitskii, Physica B+C 126, 235 (1984).

${ }^{20}$ B. L. Altshuler and A. G. Aronov, JETP Lett. 33, 499 (1981).

${ }^{21}$ P. W. Brouwer and C. W. J. Beenakker, Phys. Rev. B 55, 4695 (1997); E. McCann and I. V. Lerner, ibid. 57, 7219 (1998).

${ }^{22}$ Quantitative details and an alternative approach based on the kinetic equation will be presented elsewhere.

${ }^{23}$ Closed trajectories with $n \neq 0$ contribute only weakly to $\Delta \sigma$ at $t \ll \tau_{\mathrm{Th}}$; see Eq. (11). That is why we do not include the case $n$ $\neq 0$ in Eq. (10a) (Ref. 6).

${ }^{24}$ For specular boundary scattering in a channel geometry, see C. W. J. Beenakker and H. van Houten, Phys. Rev. B 38, 3232 (1988).

${ }^{25}$ Note that $0 \mathrm{D}$ dephasing can not be observed in a network of well-connected unit cells, since trajectories are effectively not confined at $\tau_{\varphi} / \tau_{\mathrm{Th}} \gg 1$.

${ }^{26}$ J. N. Kupferschmidt and P. W. Brouwer (private communication).

${ }^{27}$ O. Yevtushenko, G. Lutjering, D. Weiss, and K. Richter, Phys. Rev. Lett. 84, 542 (2000). 